\title{
Percepción del alumnado universitario de Educación Física sobre su competencia comunicativa
}

\author{
José Luis Gallego Ortega* \\ Antonio Rodríguez Fuentes**
}

\begin{abstract}
Resumen: Se presenta una investigación cuyo principal objetivo fue conocer las competencias comunicativas de futuros docentes de Educación Física. Se empleó un cuestionario ad hoc, que se aplicó a alumnos de primer y tercer curso de Magisterio, para valorar el progreso en dimensiones esenciales de la destreza comunicativa docente. Obtenidos los datos mediante un diseño transversal de investigación se realizaron análisis cuantitativos de tipo descriptivo. Los resultados muestran un avance general en la competencia comunicativa global de los alumnos de tercer curso con respecto a los de primero, pero que se antoja insuficiente para desempeñar las exigencias comunicativas del puesto docente.
\end{abstract}

Palabras-clave: Comunicación. Competencia profesional. Educación física.

\section{INTRODUCCIÓN}

El proceso de convergencia en el que se hallan inmersos los sistemas educativos europeos desde la Declaración de la Sorbona, en 1998, y la Declaración de Bolonia, en 1999, con las que se inicia la creación del Espacio Europeo de Educación Superior (EEES), introduce los nuevos ejes en torno a los cuales girarán los sistemas educativos de los estados miembros: las competencias y habilidades que deberán alcanzar los estudiantes cuando concluyan su formación. Así, uno de los principales retos a los que habrán de enfrentarse los futuros sistemas formativos será el de mejorar la formación inicial de los profesionales.

\footnotetext{
*Facultad de Ciencias de la Educación. Universidad de Granada. Granada, España. E-mail: jlgalleg@ugr.es

${ }^{* *}$ Facultad de Ciencias de la Educación. .Universidad de Granada. Granada, España. E-mail: arfuente@ugr.es
} 
En este contexto, la gestión del aula como espacio de comunicación y relación interpersonal se postula como una de las principales funciones del profesor eficaz, a sabiendas de que el intercambio comunicativo se percibe como la piedra angular sobre la que se construyen los conocimientos (MÉRIDA, 2013). Pero si el conocimiento y la práctica de recursos comunicativos verbales y no verbales son fundamentales para el desempeño profesional (SANZ, 2005), sin embargo muchos docentes tienen problemas para comunicar o comunicarse (CAMACHO; SÁENZ, 2000), aunque su profesionalidad dependa en buena medida de sus habilidades comunicativas.

En efecto, en los diferentes diseños de competencias que emergen como un objetivo fundamental de la formación universitaria, se subraya, entre otras claves profesionales, la figura del docente como mediador y promotor de situaciones de aprendizaje en las que son fundamentales la interrelación, la interdependencia, la colaboración y la tutorización. Sin duda se trata de una profesión de trabajo colaborativo, con un fuerte componente social e interactivo, en la que se enfatizan las competencias comunicativas, en tanto que conocimiento que nos permite utilizar el lenguaje como instrumento de comunicación en un contexto social determinado. Esta consideración se ha observado en Escocia (INITIAL...,2000), Québec (GOUVERNEMENT.., 2001; MARTINET; RAYMOND; GAUTHIER, 2004; GAUTHIER, 2006) o Chile (DANIELSON, 2011), entre otros.

En opinión de Imbernón (2007), el valor de la competencia comunicativa en la formación inicial de los Maestros es indiscutible. No obstante, el estudio de la comunicación como competencia docente es un campo relativamente nuevo. En el ámbito educativo, la mayoría de reflexiones y experiencias investigadoras cuyo tema central es la comunicación son de corte tradicional o funcionalista (BARRÓN, 1989). Se trata por tanto de experiencias que prestan atención a los aspectos mensurables del acto comunicativo (gestos, estructura y gramaticalidad del discurso, turnos comunicativos, etc.), sin duda importantes, pero descuidan el conocimiento

Movimento, Porto Alegre, v. 20, n. 2, p. 425-444, abr.jun. de 2014. 
del acto comunicativo desde un enfoque integrador, holístico, que indague sobre las concepciones o creencias de los futuros docentes en cuanto a su grado de competencia en relación con las dimensiones fundamentales para su interacción didáctica en el aula y en el centro escolar. Por consiguiente, la asunción del enfoque comunicativo representa una apertura a posiciones más profundas, proyectadas al logro de una funcionalidad y actuación satisfactoria de los estudiantes.

Más concretamente, la competencia comunicativa, que incluye procesos lingüísticos, sociolingüísticos y textuales (LOMAS; OSORO; TUSÓN, 2003), se entiende como "una síntesis singular de habilidades y estilos que se emplean acertadamente por el sujeto en consonancia con las características y exigencias de los participantes y contextos donde tiene lugar la comunicación" (DE LA UZ et al., 2010, p. 319-320), e interesa como factor de motivación, como base para la toma de decisiones y como instrumento para el mantenimiento de un clima positivo en la comunidad educativa (TEJADA, 2005). Dicha competencia incluye el dominio de distintos procesos (de codificación y descodificación) y su aplicación a diferentes hablantes y contextos (GARCÍA RUIZ, 2006). Se trata, en definitiva, de una competencia inicial básica que todo docente debería poseer (PERRENOUD, 2004), por lo que a la educación superior le corresponde garantizar la eficiente preparación del futuro docente para satisfacer las exigencias y necesidades de la enseñanza en el entorno europeo (SCOTTISH OFFICE, 2005).

Sin embargo, las competencias comunicativas, a pesar de ser valoradas en los puestos más altos de los listados de competencias transversales que se documentan en los Libros Blancos para las titulaciones del nuevo EEES, han estado durante mucho tiempo bastante desatendidas, aunque desde hace algunas décadas se resalta la trascendencia de la comunicación educativa y la necesidad de que ésta sea adecuada en el contexto escolar, por su incidencia evidente en la calidad de la enseñanza. Tal es su importancia que proliferan los estudios sobre las competencias docentes con el fin

Movimento, Porto Alegre, v. 20, n. 2, p. 425-444, abr.jun. de 2014. 
de iluminar la formación inicial del profesorado (BARRÓN, 1989; CAMACHO; MARTÍNEZ; MENDÍAS, 1999; LASNIER, 2000; CAMACHO; SÁENZ, 2000; AGENCIA..., 2005; PERRENOUD, 2004; DÍAZ BARRIGA, 2006; CASTELLÁ et al., 2007; IMBERNÓN, 2007; RICOY; FELIZ; SEVILLANO, 2010). En estos estudios se subraya unánimemente el valor de la competencia comunicativa para el desempeño docente, vinculándola con el dominio de diferentes habilidades. Se pretende así evitar el empleo de la propia intuición y valerse únicamente de la experiencia profesional para tratar de promover y desarrollar las competencias comunicativas de los estudiantes.

Teniendo en cuenta lo anterior, y con el deseo de lograr un profesional apto para realizar una labor docente de calidad, surge un interrogante de extraordinario interés: ¿los estudiantes de Educación Física (en adelante EF) poseen la competencia comunicativa necesaria para responder a las necesidades de comunicación propias de su futuro desempeño profesional?

A este efecto se realizó un estudio preliminar sobre la competencia comunicativa en estudiantes de EF, futuros Maestros de EF, durante su formación inicial. Para ello se plantearon los siguientes objetivos: 1) Conocer la percepción que poseen los estudiantes universitarios de EF acerca de sus habilidades comunicativas; 2) Establecer posibles diferencias entre los estudiantes de nuevo ingreso y quienes están próximos a egresar; 3 ) Proponer estrategias para optimizar el desarrollo de la competencia comunicativa en la formación inicial de futuros Maestros de EF.

\section{Metodología}

De acuerdo con las intenciones y el enfoque de investigación adoptado, se ha seleccionado como estrategia principal de investigación la denominada "investigación social mediante encuesta” (CEA D'ANCONA, 2001; COHEN; MANION, 2002; MCMILLAN; SCHUMACHER, 2005). Esta estrategia consiste en formular preguntas directamente a una muestra representativa

Movimento, Porto Alegre, v. 20, n. 2, p. 425-444, abr./jun. de 2014. 
de sujetos de una población concreta, para obtener datos a partir de sus declaraciones verbales (SCHUTT, 2001). Mediante un diseño transversal se realizó un análisis extensivo acerca de la percepción que tienen los estudiantes universitarios de EF sobre su competencia comunicativa en diferentes dimensiones (Cfr. Tabla 2), de acuerdo con la literatura consultada.

Se ha considerado que el análisis cuantitativo es una estrategia adecuada para resolver los objetivos formulados.

\subsection{PoBlACIÓN Y MUESTRA}

La población está formada por los estudiantes de $1^{\circ}$ y $3^{\circ}$ curso de la especialidad de EF de la Facultad de Ciencias de la Educación de la Universidad de Granada (España), que cursan la carrera de Magisterio de tres cursos académicos de duración (el nuevo Plan de Estudios, iniciado en 2010-2011, amplía un curso académico más dicha carrera).

Para la obtención de una muestra significativa, se realizó un muestreo estratificado con afijación simple o no proporcional y selección aleatoria de los sujetos a encuestar dentro de cada estrato (COHEN; MANION, 2002). El criterio de agrupamiento o estratificación ha sido la especialidad de Magisterio cursada (en nuestro caso, los Maestros de EF en formación). El tamaño muestral obtenido permite estimar proporciones con un intervalo de confianza del 95\% y un error máximo admisible del 5\%, según las tablas de Tagliacarne (1968).

La Tabla 1 contiene el tamaño de la subpoblación y submuestra, así como el total significativo y real, teniendo en cuenta:

- Subpoblación: número total de estudiantes reales asistentes a clase con regularidad;

- Submuestra estadística: datos que nos ofrece la fórmula de Tagliacarne como estadísticamente significativa para cada subpoblación (por curso y especialidad);

Movimento, Porto Alegre, v. 20, n. 2, p. 425-444, abr.jun. de 2014. 
- Submuestra real: población real de estudiantes que responden en cada uno de los estratos.

Tabla 1: Tamaños de la subpoblación y submuestra

\begin{tabular}{cccccccc}
\hline \multirow{2}{*}{ ESPECIALIDAD } & \multicolumn{2}{c}{ Subpoblación } & \multicolumn{2}{c}{$\begin{array}{c}\text { Submuestra } \\
\text { estadística }\end{array}$} & \multicolumn{2}{c}{ Submuestra real } \\
\cline { 2 - 7 } & $1^{\mathrm{o}}$ & $3^{\mathrm{o}}$ & $1^{\mathrm{o}}$ & $3^{\mathrm{o}}$ & $1^{\mathrm{o}}$ & $3^{\mathrm{o}}$ \\
\hline Educación Física & 138 & 249 & 38 & 53 & 116 & 38 \\
\hline
\end{tabular}

Fuente: De los autores

\subsection{OBTENCIÓN Y ANÁLISIS DE DATOS}

Para la obtención de datos, se recurrió al cuestionario, por tratarse de un instrumento básico para la recogida de información en la investigación mediante encuesta (SCHUTT, 2001; COHEN; MANION, 2002). Se diseñó un cuestionario ad hoc para esta investigación, con la intención de recabar datos de manera sistemática y ordenada (McMILLAN; SCHUMACHER, 2005). Inicialmente, este cuestionario fue validado por el procedimiento de "juicio de expertos" y "triangulación" (FOX, 1981; HODDER, 2000). Es decir, tres investigadores expertos en comunicación oral determinaron la validez de su contenido, de acuerdo con criterios de representatividad y relevancia de los ítem.

Posteriormente, el cuestionario se validó de forma estadística, comprobando su fiabilidad y consistencia interna. En efecto, el cuestionario obtuvo un coeficiente de fiabilidad alpha de $0,936 \mathrm{y}$ el coeficiente de fiabilidad de los ítem analizados osciló entre 0,935 y 0,936. Además, para asegurar más aún la fiabilidad del instrumento, se le aplicaron otras pruebas: prueba de las dos mitades (valor de $\alpha$ para la primera parte: 0,900 ; valor de $\alpha$ para la segunda parte: 0,881; coeficiente de Spearman-Brown: 0,866), prueba KMO (índice significativo de 0,91 , próximo a la unidad) y prueba de Bartlett (Chi-Cuadrado de 19313,955 y muy alta significación ( $\mathrm{p}=$ 0,000), obteniéndose un nivel elevado de confiabilidad.

Movimento, Porto Alegre, v. 20, n. 2, p. 425-444, abr./jun. de 2014. 
Este cuestionario incluye 5 bloques temáticos (Cfr. Tabla 2) y consta de 60 ítem, cuyas preguntas son de elección múltiple (tipo Likert) con cinco opciones en abanico o de estimación para solicitar respuestas en las que se han establecido grados de intensidad a la hora de valorar un hecho o situación: 1. Dominio nulo; 2 . Dominio insuficiente; 3. Dominio suficiente; 4. Dominio elevado; 5. Dominio muy elevado. Estas alternativas se ofrecen a los estudiantes para determinar en qué medida se consideran preparados en aspectos relacionados con la comunicación educativa, finalidad última de este estudio.

Tabla 2: Cuestionario de estudiantes

\begin{tabular}{ccc}
\hline Bloques & Competencias & Ítem \\
\hline $1^{\circ}$ & Como buen emisor & 12 \\
$2^{\circ}$ & Como buen receptor & 10 \\
$3^{\circ}$ & Habilidades comunicativas en clase & 20 \\
$4^{\text {o }}$ & Para actuar en reuniones con padres y colegas & 12 \\
$5^{\circ}$ & Para ejercer la función tutorial & 6 \\
\hline
\end{tabular}

Fuente: De los autores

El estudio de los datos cuantitativos se realizó con el Programa SPSS 15.0.

\section{Resultados}

Para una mejor comprensión de los datos, conviene señalar inicialmente la prevalencia del género femenino frente al masculino en esta Facultad de Educación (80'7\% de mujeres, 19'0\% de hombres y un 0 ' $2 \%$ que no se ha definido). De ellos, cursan la especialidad de EF el 17'3\%.

Del alumnado escolarizado en $1^{\circ}$, un $79^{\prime} 4 \%$ son mujeres frente al 20 ' $4 \%$ de hombres y un 0 ' $2 \%$ de la muestra no se identifica. Cursan estudios de EF un 22'3 \%. Asimismo, del alumnado escolarizado en

Movimento, Porto Alegre, v. 20, n. 2, p. 425-444, abr.jun. de 2014. 
$3^{\circ}$ curso, un $16^{\prime} 9 \%$ son varones, un $82{ }^{\prime} 3 \%$ mujeres y un $0{ }^{\prime} 5 \%$ no se definen. De ellos, realizan los estudios de EF un 10’2\%.

A continuación, se sintetizan los datos y se señalan los porcentajes, tanto en la especialidad (EF) como por curso $\left(1^{\circ} \mathrm{y}\right.$ $3^{\circ}$ ), teniendo en cuenta que los valores 1,00 y 5,00 representan la percepción más baja/alta de dominio de las habilidades comunicativas. No obstante, para la correcta interpretación de los datos, se han tenido en cuenta conjuntamente los valores 1 y 2 , que representan la falta de dominio de la capacidad, y los valores 3 y 4 , que indican un dominio de la capacidad.

\subsection{EN EL ANÁLISIS DE CONTINGENCIAS SOBRE PERCEPCIÓN GENERAL}

En los ítem que se detallan en la Tabla 3, el alumnado de EF admite, aunque con diferente porcentaje, una evidente preferencia por el valor 3, que representa un dominio suficiente de las habilidades comunicativas señaladas.

Tabla 3: Ítem con valor 3

\begin{tabular}{ll}
\hline V2 & me expreso por escrito con propiedad y corrección \\
\hline V3 & pienso lo que voy a decir antes de expresarlo \\
\hline V6 & me siento seguro cuando tengo que mantener una conversación \\
\hline V7 & contrasto opiniones antes de imponer mis propias ideas directamente \\
\hline V8 & $\begin{array}{l}\text { evito que la opinión que me merece el receptor reste objetividad a mi } \\
\text { condición de emisor }\end{array}$ \\
\hline V10 & utilizo un lenguaje comprensible en mis relaciones con los demás \\
\hline V11 & permito que quien me escucha me manifieste hasta qué punto me entiende \\
\hline V12 & sobre aspectos generales acerca de la habilidad de buen emisor \\
\hline V13 & respeto las opiniones o ideas de quien me habla \\
\hline V14 & me concentro en escuchar antes de emitir un juicio u opinión \\
\hline V15 & me pongo en el lugar del otro para comprender mejor sus reacciones \\
\hline V16 & me cercioro de que he comprendido antes de intervenir \\
\hline V18 & descubro fácilmente el hilo conductor de lo que se me dice \\
\hline V19 & presto atención al contexto para entender el significado de las palabras \\
\hline
\end{tabular}

Movimento, Porto Alegre, v. 20, n. 2, p. 425-444, abr./jun. de 2014. 


\begin{tabular}{|c|c|}
\hline V20 & conservo la calma aunque el que me hable está excitado \\
\hline $\mathrm{V} 21$ & hago preguntas para cerciorarme de que he comprendido bien \\
\hline V22 & $\begin{array}{l}\text { en general, considero que mi dominio de las habilidades de buen receptor } \\
\text { son }\end{array}$ \\
\hline V23 & articular clara y correctamente y a la velocidad moderada \\
\hline V28 & captar y mantener la atención de los alumnos \\
\hline V29 & crear en el aula un clima de relación positivo \\
\hline $\mathrm{V} 30$ & hacer uso de distintas modalidades de preguntas \\
\hline V32 & utilizar productivamente el sentido del humor \\
\hline V33 & aplicar técnicas de trabajo en pequeños grupos \\
\hline V34 & conocer los contenidos que voy a impartir \\
\hline V35 & afrontar las situaciones comprometidas en clase \\
\hline V38 & sentirme seguro para conducir el grupo clase \\
\hline V39 & $\begin{array}{l}\text { utilizar los gestos y otras expresiones corporales para mejorar la } \\
\text { comunicación }\end{array}$ \\
\hline V40 & utilizar un lenguaje comprensible en las exposiciones en clase \\
\hline V42 & $\begin{array}{l}\text { en general, considero que mi dominio de los aspectos comunicativos de } \\
\text { la labor docente son }\end{array}$ \\
\hline V43 & prestar atención a lo que dicen los demás \\
\hline V46 & centrarme en la materia objeto de debate \\
\hline V47 & $\begin{array}{l}\text { respetar las opiniones de los otros miembros del grupo y sus turnos de } \\
\text { intervención }\end{array}$ \\
\hline V49 & expresarme con concisión, claridad y precisión \\
\hline V50 & $\begin{array}{l}\text { esforzarme para que mis intervenciones sean percibidas por los demás } \\
\text { como una contribución importante al logro de los objetivos del grupo }\end{array}$ \\
\hline V52 & $\begin{array}{l}\text { acompañar mis críticas con sugerencias que contribuyan a solucionar el } \\
\text { problema }\end{array}$ \\
\hline V53 & $\begin{array}{l}\text { respetar las decisiones acordadas por la mayoría de los miembros aunque } \\
\text { no coincidan con nuestras propias posiciones }\end{array}$ \\
\hline V54 & $\begin{array}{l}\text { en general, considero que mi dominio de las habilidades propias del } \\
\text { participante eficaz en reuniones es bueno }\end{array}$ \\
\hline V57 & evaluar y registrar los resultados de la entrevista \\
\hline V59 & $\begin{array}{l}\text { interpretar el significado de los signos verbales y no verbales durante la } \\
\text { entrevista }\end{array}$ \\
\hline
\end{tabular}

Fuente: De los autores 
Por el contrario, los ítems en los que los estudiantes universitarios de EF optan, en mayor medida y en porcentaje diferente por el valor 2 , que se caracteriza por aludir a una percepción de dominio insuficiente de las habilidades comunicativas, son los siguientes (Cfr. Tabla 4).

Tabla 4: Ítem con valor 2

\begin{tabular}{cl}
\hline V1 & me expreso oralmente con propiedad y corrección \\
\hline V4 & persuado a los demás con mis argumentos \\
\hline V9 & evito emitir opiniones categóricas sobre asuntos que ignoro \\
\hline V12 & sobre aspectos generales acerca de la habilidad de buen emisor \\
\hline V17 & prescindo de los prejuicios que tengo acerca de quien me habla \\
\hline V24 & organizar adecuadamente mis exposiciones \\
\hline V26 & $\begin{array}{l}\text { aplicar estrategias de motivación para interesar a los alumnos en la } \\
\text { materia o materias }\end{array}$ \\
\hline V27 & usar ejemplos adecuados a los contenidos y al nivel de los alumnos \\
\hline V36 & lograr que los alumnos se centren en la tarea \\
\hline V37 & utilizar productivamente la crítica y la alabanza \\
\hline V41 & $\begin{array}{l}\text { permitir que los alumnos manifiesten sus dudas sobre lo explicado o } \\
\text { realizado }\end{array}$ \\
\hline V44 & autorregular la frecuencia y la duración de mis propias intervenciones \\
\hline V45 & preparar con antelación los puntos fuertes de mis argumentaciones \\
\hline V48 & intervenir con naturalidad y sin inhibiciones \\
\hline V51 & $\begin{array}{l}\text { mantener en mis intervenciones un tono mesurado, cortés y } \\
\text { constructivo }\end{array}$ \\
\hline V55 & planificar sistemáticamente la entrevista de tutoría \\
\hline V56 & aplicar las técnicas que requiere la relación de ayuda \\
\hline V58 & crear un ambiente propicio a la relación interpersonal \\
\hline V60 & $\begin{array}{l}\text { en general, considero que mi dominio de las habilidades comunicativas } \\
\text { propias del profesor tutor son }\end{array}$ \\
\hline &
\end{tabular}

Fuente: De los autores

Sin embargo, en el ítem 25 ("utilizar un nivel de vocabulario adecuado") los estudiantes se muestran dubitativos, es decir, tienen dificultades para decantarse entre un grado de dominio suficiente

Movimento, Porto Alegre, v. 20, n. 2, p. 425-444, abr./jun. de 2014. 
Percepción del alumnado universitario de Educación Física ...

(v3) o insuficiente (v2), lo cual indica una indefinición a la hora de estimar cuál es verdaderamente el grado de dominio que admiten poseer de esta habilidad comunicativa. Únicamente el ítem 5 ("me siento seguro cuando tengo que iniciar una comunicación") concentra el menor número de respuestas preferentes de los estudiantes en el valor 1, lo que muestra un nivel de percepción nulo de esta habilidad comunicativa. Es más, la suma de los valores 1 y 2 , en ambos cursos, supera el 60\% de las respuestas.

En la Tabla 5 se incluyen los valores preferentes seleccionados por los estudiantes de EF y sus porcentajes concretos.

Tabla 5: Valores preferidos y porcentajes de la especialidad

\begin{tabular}{ccccccccccccc}
\hline Ítem & $\mathbf{1}$ & $\mathbf{2}$ & $\mathbf{3}$ & $\mathbf{4}$ & $\mathbf{5}$ & $\mathbf{6}$ & $\mathbf{7}$ & $\mathbf{8}$ & $\mathbf{9}$ & $\mathbf{1 0}$ & $\mathbf{1 1}$ & $\mathbf{1 2}$ \\
\hline Valores & $\mathrm{V} 2$ & $\mathrm{~V} 3$ & $\mathrm{~V} 3$ & $\mathrm{~V} 2$ & $\mathrm{~V} 1$ & $\mathrm{~V} 3$ & $\mathrm{~V} 3$ & $\mathrm{~V} 3$ & $\mathrm{~V} 2$ & $\mathrm{~V} 3$ & $\mathrm{~V} 3$ & $\mathrm{~V} 3$ \\
\cline { 2 - 10 }$\%$ & 40.3 & 58.4 & 51.9 & 45.5 & 32.5 & 45.5 & 40.3 & 39.0 & 48.7 & 56.5 & 38.3 & 44.2 \\
\hline Ítem & $\mathbf{1 3}$ & $\mathbf{1 4}$ & $\mathbf{1 5}$ & $\mathbf{1 6}$ & $\mathbf{1 7}$ & $\mathbf{1 8}$ & $\mathbf{1 9}$ & $\mathbf{2 0}$ & $\mathbf{2 1}$ & $\mathbf{2 2}$ & $\mathbf{2 3}$ & $\mathbf{2 4}$ \\
\hline Valores & $\mathrm{V} 3$ & $\mathrm{~V} 3$ & $\mathrm{~V} 3$ & $\mathrm{~V} 3$ & $\mathrm{~V} 2$ & $\mathrm{~V} 3$ & $\mathrm{~V} 3$ & $\mathrm{~V} 3$ & $\mathrm{~V} 3$ & $\mathrm{~V} 3$ & $\mathrm{~V} 3$ & $\mathrm{~V} 2$ \\
\cline { 2 - 11 }$\%$ & 33.8 & 46.8 & 48.1 & 46.8 & 42.2 & 61.0 & 44.8 & 44.2 & 42.2 & 62.3 & 44.2 & 57.8 \\
\hline Ítem & $\mathbf{2 5}$ & $\mathbf{2 6}$ & $\mathbf{2 7}$ & $\mathbf{2 8}$ & $\mathbf{2 9}$ & $\mathbf{3 0}$ & $\mathbf{3 1}$ & $\mathbf{3 2}$ & $\mathbf{3 3}$ & $\mathbf{3 4}$ & $\mathbf{3 5}$ & $\mathbf{3 6}$ \\
\hline Valores & $\mathrm{V} 2 / 3$ & $\mathrm{~V} 2$ & $\mathrm{~V} 2$ & $\mathrm{~V} 3$ & $\mathrm{~V} 3$ & $\mathrm{~V} 3$ & $\mathrm{~V} 2$ & $\mathrm{~V} 3$ & $\mathrm{~V} 3$ & $\mathrm{~V} 3$ & $\mathrm{~V} 3$ & $\mathrm{~V} 2$ \\
\cline { 2 - 11 }$\%$ & 46.4 & 43.5 & 38.3 & 44.2 & 41.6 & 39.0 & 41.6 & 47.4 & 46.1 & 50.0 & 47.4 & 48.7 \\
\hline Ítem & $\mathbf{3 7}$ & $\mathbf{3 8}$ & $\mathbf{3 9}$ & $\mathbf{4 0}$ & $\mathbf{4 1}$ & $\mathbf{4 2}$ & $\mathbf{4 3}$ & $\mathbf{4 4}$ & $\mathbf{4 5}$ & $\mathbf{4 6}$ & $\mathbf{4 7}$ & $\mathbf{4 8}$ \\
\hline Valores & $\mathrm{V} 2$ & $\mathrm{~V} 3$ & $\mathrm{~V} 3$ & $\mathrm{~V} 3$ & $\mathrm{~V} 2$ & $\mathrm{~V} 3$ & $\mathrm{~V} 3$ & $\mathrm{~V} 2$ & $\mathrm{~V} 2$ & $\mathrm{~V} 3$ & $\mathrm{~V} 3$ & $\mathrm{~V} 2$ \\
\cline { 2 - 11 }$\%$ & 44.2 & 42.9 & 38.3 & 39.0 & 42.9 & 43.5 & 39.0 & 49.4 & 37.0 & 47.4 & 53.9 & 38.3 \\
\hline Ítem & $\mathbf{4 9}$ & $\mathbf{5 0}$ & $\mathbf{5 1}$ & $\mathbf{5 2}$ & $\mathbf{5 3}$ & $\mathbf{5 4}$ & $\mathbf{5 5}$ & $\mathbf{5 6}$ & $\mathbf{5 7}$ & $\mathbf{5 8}$ & $\mathbf{5 9}$ & $\mathbf{6 0}$ \\
\hline Valores & $\mathrm{V} 3$ & $\mathrm{~V} 3$ & $\mathrm{~V} 2$ & $\mathrm{~V} 3$ & $\mathrm{~V} 3$ & $\mathrm{~V} 3$ & $\mathrm{~V} 2$ & $\mathrm{~V} 2$ & $\mathrm{~V} 3$ & $\mathrm{~V} 2$ & $\mathrm{~V} 3$ & $\mathrm{~V} 2$ \\
\cline { 2 - 10 }$\%$ & 49.4 & 45.5 & 42.9 & 48.1 & 43.5 & 43.5 & 43.5 & 42.2 & 44.2 & 40.3 & 51.3 & 46.8 \\
\hline
\end{tabular}

Fuente: De los autores

3.2 EN EL ANÁLISIS DE CONTINGENCIAS POR CURSO

En un primer grupo de variables de contingencias por cursos, se han reunido todos los ítem (v1, v12, v17, v20, v21, v23, v24, v25,

Movimento, Porto Alegre, v. 20, n. 2, p. 425-444, abr./jun. de 2014. 
v26, v27, v28, v30, v31, v33,v37, v38, v39, v40, v41, v42, v44, v46, v48, v49, v50, v51, v52, v53, v54, v55, v56, v58, v59, v60) que obtienen una puntuación con tendencia mayoritaria. En este sentido, se aprecia que los estudiantes de $1^{\circ}$ optan mayoritariamente por el valor 2 , que representa en el cuestionario un nivel de dominio insuficiente de estas competencias comunicativas. Sin embargo, la mayoría de los estudiantes de 3er curso, en los ítem señalados, se muestra favorable a elegir el valor 3, que supone un dominio suficiente de la habilidad comunicativa correspondiente. De estos resultados puede inferirse un incremento de las habilidades comunicativas durante la carrera, según la percepción de los propios estudiantes.

En un segundo grupo de variables (v2, v3, v10, v11, v15, v16, v18, v19, v22, v29, v32, v43, v45, v47), todos los estudiantes (tanto de $1^{\circ}$ como de $3^{\circ}$ ) se decantan en mayor medida, aunque con porcentajes diferentes, por el valor 3 , que indica un dominio suficiente de estas competencias. Si bien, porcentualmente, hay diferencias entre ambos grupos siempre a favor de los estudiantes de 3 er curso. Es un bloque de competencias en las que se ha progresado, pero muy levemente.

En un tercer grupo de variables (v4, v5, v8, v35, v36, v57), se puede observar tendencias comunes entre los estudiantes de $1^{\circ}$ y los de $3^{\circ}$, al concentrarse las respuestas de los estudiantes en el valor 2 (dominio insuficiente).

En cuarto lugar, que incluye un solo ítem (v6: me siento seguro cuando tengo que mantener una conversación"), se constata que el alumnado de $1^{\circ}$ tiene una valoración más alta (dominio suficiente) que sus compañeros de $3^{\circ}$, respecto a su competencia en esta capacidad (dominio insuficiente). Ello podría deberse a un incremento de su consciencia sobre lo que supone ser competente en esta habilidad conversacional.

Finalmente, en el quinto grupo de variables (v13 v14) se aprecia que el alumnado de ambos cursos posee una percepción elevada sobre estas competencias comunicativas. En efecto, los estudiantes

Movimento, Porto Alegre, v. 20, n. 2, p. 425-444, abr.jun. de 2014. 
Percepción del alumnado universitario de Educación Física ...

de $1^{\circ}$ optan preferentemente por el valor 3 (dominio suficiente) y los de $3^{\circ}$, por su parte, valoran aún más estas habilidades, señalando mayoritariamente el valor 4 (dominio elevado).

En la Tabla 6 se incluyen los valores preferentes y porcentajes por cursos $\left(1^{\circ}\right.$ y $\left.3^{\circ}\right)$.

Tabla 6: Valores preferidos y porcentajes por cursos $\left(1^{\circ}\right.$ y $\left.3^{\circ}\right)$

\begin{tabular}{|c|c|c|c|c|c|c|c|c|c|c|c|c|}
\hline Ítem & 1 & 2 & 3 & 4 & 5 & 6 & 7 & 8 & 9 & 10 & 11 & 12 \\
\hline Valores & v $2 / 3$ & v3 & v3 & v2 & v2 & $\mathrm{v} 3 / 2$ & v2 & v2 & $v 2 / 3$ & v3 & v3 & $\mathrm{v} 2 / 3$ \\
\hline$(\%$ & 55.1 & 44.5 & 41.8 & 50.9 & 33.3 & 40.8 & 40.5 & 46.6 & 36.2 & 52.2 & 43.9 & 49.7 \\
\hline $\left.1^{\circ} / 3^{\circ}\right)$ & 40.4 & 39.9 & 38 & 37.5 & 41.0 & 36.7 & 35.8 & 40.4 & 35.8 & 43,7 & 45.3 & 40.4 \\
\hline Ítem & 13 & 14 & 15 & 16 & 17 & 18 & 19 & 20 & 21 & 22 & 23 & 24 \\
\hline Valores & v3/4 & v $3 / 4$ & v3 & v3 & $\mathrm{v} 2 / 3$ & v3 & v3 & $\mathrm{v} 2 / 3$ & $\mathrm{v} 2 / 3$ & v3 & v $2 / 3$ & $\mathrm{v} 2 / 3$ \\
\hline$(\%$ & 33.9 & 43 & 42.2 & 44.1 & 55.5 & 49.1 & 45.1 & 40.1 & 43.7 & 48.7 & 54.5 & 53.6 \\
\hline $\left.1^{\circ} / 3^{\circ}\right)$ & 47.4 & 41.8 & 44.2 & 43.7 & 42.9 & 39.1 & 43.4 & 36.1 & 39.1 & 41.2 & 48 & 45 \\
\hline Ítem & 25 & 26 & 27 & 28 & 29 & 30 & 31 & 32 & 33 & 34 & 35 & 36 \\
\hline Valores & $\mathrm{v} 2 / 3$ & $\mathrm{v} 2 / 3$ & $\mathrm{v} 2 / 3$ & $\mathrm{v} 2 / 3$ & v3 & $\mathrm{v} 2 / 3$ & $\mathrm{v} 2 / 3$ & v3 & $\mathrm{v} 2 / 3$ & v3 & v2 & v2 \\
\hline$(\%$ & 54.3 & 39.3 & 42.8 & 41.6 & 40.5 & 48.7 & 42.6 & 41.6 & 40.5 & 40.1 & 40.8 & 47.8 \\
\hline $\left.1^{\circ} / 3^{\circ}\right)$ & 43.7 & 37.5 & 37.7 & 39.4 & 36.4 & 40.4 & 38.8 & 34.5 & 44.7 & 41 & 34.5 & 36.1 \\
\hline Ítem & 37 & 38 & 39 & 40 & 41 & 42 & 43 & 44 & 45 & 46 & 47 & 48 \\
\hline Valores & $\mathrm{v} 2 / 3$ & $\mathrm{v} 2 / 3$ & $\mathrm{v} 2 / 3$ & $\mathrm{v} 2 / 3$ & $\mathrm{v} 2 / 3$ & $\mathrm{v} 2 / 3$ & v3 & $\mathrm{v} 2 / 3$ & v3 & $\mathrm{v} 2 / 3$ & v3 & $\mathrm{v} 2 / 3$ \\
\hline$(\%$ & 49.5 & 36.8 & 42.6 & 43.9 & 34.1 & 50.3 & 41.2 & 58.2 & 37.6 & 46.4 & 38 & 46.4 \\
\hline $\left.1^{\circ} / 3^{\circ}\right)$ & 40.2 & 35.8 & 32.6 & 39.9 & 36.7 & 41.8 & 38.3 & 36.1 & 42.6 & 45.3 & 36.1 & 35 \\
\hline Ítem & 49 & 50 & 51 & 52 & 53 & 54 & 55 & 56 & 57 & 58 & 59 & 60 \\
\hline Valores & v $2 / 3$ & $\mathrm{v} 2 / 3$ & $\mathrm{v} 2 / 3$ & $\mathrm{v} 2 / 3$ & $\mathrm{v} 2 / 3$ & $\mathrm{v} 2 / 3$ & $\mathrm{v} 2 / 3$ & $\mathrm{v} 2 / 3$ & v2 & $\mathrm{v} 2 / 3$ & v $2 / 3$ & $\mathrm{v} 2 / 3$ \\
\hline$(\%$ & 40.8 & 50.5 & 48.2 & 43 & 44.1 & 49.7 & 45.1 & 49.9 & 45.1 & 50.7 & 43.2 & 54.5 \\
\hline $\left.1^{\circ} / 3^{\circ}\right)$ & 41.8 & 39.1 & 43.9 & 44.2 & 40.7 & 46.4 & 36.4 & 38.8 & 35.8 & 38.3 & 37.2 & 40.4 \\
\hline
\end{tabular}

Fuente: De los autores

\section{Discusión Y CONCLUSIONES}

Con anterioridad, se ha presentado una síntesis de los resultados y porcentajes obtenidos en este estudio. Esta es la razón por la cual, al presentar las conclusiones finales, nos limitaremos a resaltar aquellas observaciones de carácter general que se desprenden del mismo, evitando la repetición de datos puntuales que pueden comprobarse en las páginas precedentes. No obstante,

Movimento, Porto Alegre, v. 20, n. 2, p. 425-444, abr./jun. de 2014. 
como se recordará, para que la valoración de los datos reflejados en las Tablas 5 y 6 no induzca a error, su apreciación última deriva de la consideración conjunta (suma de porcentajes) de los valores 1 y 2 (que representan el dominio nulo/insuficiente de la capacidad) frente a los valores 3 y 4 (que señalan el dominio suficiente/elevado de la misma).

Desde esta perspectiva, se ha de señalar inicialmente que, aunque la competencia comunicativa es considerada como una habilidad básica para el desempeño de la actividad docente (CAMACHO; SÁENZ, 2000; PERRENOUD, 2004; SANZ, 2005; CASTELLÁ et al., 2007), esta investigación nos ha permitido probar que (a) el nivel de competencia comunicativa de los Maestros de EF en formación muestra numerosas limitaciones, (b) el alumnado de $3^{\circ}$ curso de esta especialidad admite un mayor nivel de competencia comunicativa que el de $1^{\circ}$, (c) es necesario incorporar en futuros planes de formación técnicas y estrategias de comunicación eficaz que fomenten el desarrollo de la competencia comunicativa de los futuros docentes de EF.

No obstante, de manera detallada, la investigación reveló cuáles eran las percepciones que poseían los Maestros de EF en formación, en cuanto a su competencia comunicativa. En este sentido, estos estudiantes se consideran, en general, con un nivel de dominio suficiente de sus habilidades como emisores docentes, especialmente cuando deben expresarse de forma escrita con propiedad y corrección -lo cual difiere de los datos obtenidos en estudios previos (AGENCIA..., 2003; GALLEGO; GARCÍA; RODRÍGUEZ, 2013)-, mantener una conversación, contrastar opiniones, utilizar un lenguaje comprensible, escuchar a los demás o pensar antes de hablar. No obstante, como ya pusieran de manifiesto otros estudios que abordan los problemas para lograr una eficaz comunicación educativa oral (CAMACHO; SÁENZ, 2000; AGENCIA...,, 2003; NÚÑEZ; DEL MORAL, 2010), se suponen insuficientemente capacitados para expresarse oralmente con propiedad y corrección o persuadir argumentalmente a sus interlocutores.

Movimento, Porto Alegre, v. 20, n. 2, p. 425-444, abr.jun. de 2014. 
Así mismo, aunque no se sienten seguros para iniciar una comunicación, ni de prescindir de los prejuicios de sus interlocutores, piensan que sus habilidades como receptores son suficientes, ya que se sienten habilitados para respetar opiniones o ideas ajenas, emitir juicios fundados, intentar comprender mensajes y reacciones, no intervenir sin fundamento, considerar el contexto antes de manifestar una opinión o conservar la calma en ambientes adversos. Por tanto, de acuerdo con los datos obtenidos, es la habilidad en la que se encuentran más preparados los estudiantes.

De otra parte, en términos generales, sus habilidades comunicativas en el aula las estiman suficientes, a sabiendas de que una comunicación eficaz hará posible la construcción de nuevos significados y conocimientos por el alumno a partir del traslado efectivo de las diferentes propuestas docentes (VILLALOBOS, 2011). En este sentido, se consideran capaces de hablar con un lenguaje comprensible en la clase, implicar al alumnado, formular diferentes preguntas, utilizar productivamente el sentido del humor, aplicar las técnicas de trabajo, conocer los contenidos educativos, afrontar situaciones comprometidas, conducir el grupo-clase y hacer un uso adecuado de la comunicación no verbal para complementar la comunicación oral. Si bien titubean sobre su preparación para utilizar el léxico más adecuado a cada situación comunicativa y confiesan serias dudas en cuanto a sus habilidades para prescindir de prejuicios previos, organizar correctamente sus exposiciones, motivar al alumnado, manejar ejemplos oportunos y utilizar productivamente la crítica y la alabanza.

Igualmente, estos estudiantes admiten notables carencias para manejarse en reuniones relacionadas con su futura labor docente, dadas sus limitaciones en cuanto a la autorregulación de sus intervenciones o la preparación previa de sus argumentaciones, así como en la dificultad para intervenir con naturalidad y sin inhibiciones o mantener un tono mesurado, cortés y constructivo. Al contrario, suponen que tienen un dominio suficiente en otras habilidades comunicativas vinculadas con la gestión de la comunicación: expresarse con claridad, prestar atención a

Movimento, Porto Alegre, v. 20, n. 2, p. 425-444, abr.jun. de 2014. 
los demás y respetar sus opiniones y turnos de intervención, centrarse en la materia objeto de debate, esforzarse para contribuir al logro de los objetivos del grupo, acompañar sus críticas con sugerencias que contribuyan a solucionar el problema o cumplir las decisiones acordadas. Los estudios en la sala de clase evidencian que aquello que es posible aprender se aprende en interacción (EMANUELSSON; SAHLSTRON, 2008).

Además estos estudiantes perciben que su competencia como tutores es insuficiente, ya que, aunque se consideran competentes para evaluar y registrar los resultados de una entrevista o interpretar los gestos y mensajes de sus interlocutores, no se sienten capaces de crear ambientes estimulantes, recurrir a técnicas de ayuda personalizada o planificar la entrevista de una tutoría, cuando el valor de esta competencia docente en las entrevistas y comunicación con las familias y con otros colegas resulta trascendental para alcanzar una comprensión compartida de lo que constituye una buena docencia y para el diálogo profesional y educativo (DANIELSON, 2011). La comunicación es, pues, clave para el contacto entre docentes, alumnos y familias, y la tutoría, en tanto que función docente, resulta muy útil no solo para prevenir dificultades de aprendizaje sino para transitar desde modelos centrados en la enseñanza hacia modelos centrados en el aprendizaje.

Se observa, asimismo, que los sujetos de nuestra muestra manifiestan una tendencia positiva en el desarrollo de competencias comunicativas desde que inician sus estudios hasta que los concluyen. Los alumnos de $3^{\circ}$, tal vez por diversas razones, incluida la madurez, muestran mayor conciencia de competencia comunicativa que los de $1^{\circ}$. En efecto, aunque la mejora pueda tildarse de tímida, a tenor de los datos obtenidos, los alumnos de $3^{\circ}$ muestran un grado de dominio superior a los de $1^{\circ}$ en todos los bloques del cuestionario, a excepción del ítem 6 del bloque 1, que alude a la seguridad del futuro docente en formación para mantener una conversación.

Finalmente, el estudio ha puesto de manifiesto que estos estudiantes se sienten especialmente vulnerables cuando tienen

Movimento, Porto Alegre, v. 20, n. 2, p. 425-444, abr.jun. de 2014. 
que iniciar una comunicación, dada su nula o escasa capacidad de iniciativa, y a la hora de organizar adecuadamente sus exposiciones. Sin embargo, se muestran mucho más seguros cuando deben expresarse por escrito, a la hora de utilizar un lenguaje comprensible con sus interlocutores o descubrir la relación entre mensajes.

De los resultados obtenidos en este estudio, se pueden señalar algunas pautas o estrategias didácticas para mejorar las habilidades comunicativas del futuro docente: 1) incorporar de forma reglada el desarrollo de las competencias comunicativas en las futuras Guías de formación docente; 2) adecuar el discurso a cada situación comunicativa, a partir del análisis de los contextos en los que se produce la comunicación; 3) conocer y experimentar con diferentes recursos lingüísticos y no lingüísticos para mantener la atención del grupo; 4) comprender cómo se estructuran los mensajes orales; 5) conocer y manejar técnicas de dinámica de grupos.

El carácter exploratorio de esta investigación, no obstante, aconseja una interpretación prudente de los resultados, especialmente a la hora de generalizarlos, dado que están abiertos a futuras comprobaciones o réplica. Pero el carácter inicial de este estudio abre nuevos interrogantes de especial interés para la investigación en este campo.

Percepção dos estudantes universitários de Educação Física sobre as suas competências comunicativas

Resumo: A pesquisa teve como principal objetivo avaliar as habilidades de comunicação de futuros professores de educação física. Um questionário ad hoc foi aplicado aos alunos do primeiro e terceiro ano do Magistério para avaliar o progresso na habilidade comunicativa. Os dados foram analisados usando estudos transversais e análises quantitativas descritivas. Os resultados mostram um avanço geral em competência comunicativa global de alunos da terceira série sobre o primeiro, mas que parece insuficiente para atender as demandas de comunicação do cargo de professor.

Palavras-chave: Comunicação. Competência profissional. Educação Física.

Movimento, Porto Alegre, v. 20, n. 2, p. 425-444, abr.jun. de 2014. 


\begin{abstract}
Perception of university students of Physical Education about their communicative competence

Abstract: The main objective of this research was to know the communicative competences of the university students of Physical Education. It was used an "ad hoc" questionnaire which was applied to students in their first and third course of Educational Practice, in order to analyze their progress in essential areas of the teachers communicative competence. With the information obtained via a transverse design of investigation, quantitative analyses were made. The results show a general progress in the communicative global competences of the third level students compared to the first level students, although insufficient to practice the activities of the teachers.
\end{abstract}

Keywords: comunication. Professional competence. Physical education.

\title{
REFERENCIAS
}

AGENCIA NACIONAL DE EVALUACIÓN DE LA CALIDAD Y ACREDITACIÓN (ANECA). Libro Blanco del Grado en Magisterio. Madrid, 2005.

AGENCIA PARA LA CALIDAD DE SISTEMA EDUCATIVO DE CATALUÑA (AQU). Educación Superior y trabajo en Cataluña. Barcelona, 2003.

BARRÓN, Ángela. Presupuestos para un programa racional de formación del enseñante. Bordón, Madrid, n. 4, p. 681-689, 1989.

CAMACHO, Salvador; MARTÍNEZ, Francisco; MENDÍAS, Ana Ma . La comunicación en las organizaciones educativas. En: LORENZO, Manuel; ORTEGA, José Antonio; CORCHÓN, Eudaldo (Ed.). Enfoques comparados en organización y dirección de instituciones educativas. Granada: GEU, 1999. p. 297-304.

CAMACHO, Salvador; SÁEZ, Óscar. Técnicas de comunicación eficaz para profesores y formadores. Alcoy: Marfil, 2000.

CASTELLÁ, Josep; COMELLES, Salvador; CROSS, Anna; VILÁ, Montserrat Entender(se) en clase: las estrategias comunicativas de los docentes bien valorados. Barcelona: Graó, 2007.

CEA D'ANCONA, Ma Ángeles. Metodología cuantitativa: Estrategias y técnicas de investigación social. Madrid: Síntesis, 2001.

COHEN, Louis; MANION, Lawrence. Métodos de investigación educativa. Madrid: La Muralla, 2002.

DANIELSON, Charlotte. Competencias docentes: Desarrollo, apoyo y evaluación. Serie Documentos PREAL, 51. Santiago de Chile: CINDE/PREAL, 2011.

Movimento, Porto Alegre, v. 20, n. 2, p. 425-444, abr./jun. de 2014. 
DE LA UZ, M ${ }^{a}$ del Carmen; DE LA UZ, Ma Elena; LEMUS, Agustín; VALDÉS María N.; PADRÓN, Carmen I. Competencia comunicativa en los estudiantes de medicina: diagnóstico preliminar. Revista de Ciencias Médicas de Pinar del Río, Pinar del Río, v. 14, n. 1, p. 314-326, 2010. Disponible en: <http://scielo.sld.cu/pdf/ rpr/v14n1/rpr31110.pdf>. Acceso en: 12 jul. 2013.

DÍAZ BARRIGA, Ángel. El enfoque de competencias en la educación. ¿Una alternativa o un disfraz de cambio? Perfiles Educativos, Mexico, DF, v. 23, n. 111, p. 7-36, 2006.

EMANUELSSON, Jonas; SAHLSTRON, Fritjof. The Price of Participation: Teacher control versus student participation in classroom interaction. Scandinavian Journal of Educational Research, Uppsala , v. 52, n. 2, p. 205-223, 2008.

FOX, David. El proceso de investigación en educación. Pamplona: EUNSA, 1981.

GALLEGO, José Luis; GARCíA, Antonio; RODRÍGUEZ, Antonio. Cómo escriben los futuros docentes. Estrategias para la mejora. Málaga: Aljibe, 2013.

GARCÍA RUIZ, Ma Rosa. Las competencias de los alumnos universitarios. Revista Interuniversitaria de Formación del Profesorado, Zaragoza, v. 20, n. 3, p. 253269, 2006.

GAUTHIER, Clermont. La política sobre formación inicial de docentes en Québec. Revista de Educación, Madrid, n. 340, p. 165-185, 2006.

GOUVERNEMENT DU QUEBEC. MINISTERE DE L'ÉDUCATION. La formation à l'enseignement : les orientations, es compétences professionnelles. Québec, 2001.

HODDER, lan. The interpretation of documents and material culture. En: DENZIN, Norman Kent; LINCOLN, Yvonna Sessions (Ed.). Handbook of Qualitative Research. London: Sage Publications, 2000. p. 703-717.

IMBERNÓN, Francisco. La formación permanente del profesorado: Nuevas ideas para formar en la innovación y el cambio. Barcelona: Graó, 2007.

INITIAL TEACHER EDUCATION IN SCOTLAND. The Standard for Initial Teacher Education in Scotland., Quality Assurance Agency for Higher Education, 2000. Disponible en: <http://www.scotland.gob.uk>. Acceso en: 12 marzo 2012.

LASNIER, François. Réussir la formation par compétences. Montreal: Guerin, 2000.

LOMAS, Carlos; OSORO, Andrés; TUSÓN, Amparo. Ciencia del Lenguaje, Competencia Comunicativa y Enseñanza de la Lengua. Barcelona: Paidós, 2003.

MARTINET, Marielle; RAYMOND, Danielle; GAUTHIER, Clermont. Formación de docentes. Orientaciones y Competencias profesionales. Québec: Ministère de l'Education, 2004.

Movimento, Porto Alegre, v. 20, n. 2, p. 425-444, abr.jun. de 2014. 
McMILLAN, James; SCHUMACHER, Sally. Investigación educativa: una introducción conceptual, Madrid: Pearson Addison Wesley, 2005.

MÉRIDA, Rosario. La controvertida aplicación de las competencias en la formación docente universitaria. Revista de Docencia Universitaria. REDU, Santiago de Compostela, v. 11, n. 1, p. 185-212, 2013.

NÚÑEZ, Ma Pilar; DEL MORAL, Cristina. Competencia léxica y competencia comunicativa: bases para el diseño de programas didácticos en la educación escolar. Lenguaje y Textos, Madrid, n. 1, p. 91-97, 2010.

PERRENOUD, Philippe. Diez nuevas competencias para enseñar. Barcelona: Graó, 2004.

RICOY, Ma Carmen; FELIZ, Tiberio; SEVILLANO, M ${ }^{a}$ Luisa. Competencias para la utilización de las herramientas digitales en la sociedad de la información. Educación XX1, Madrid, v. 13, n. 1, p. 199-219, 2010.

SANZ, Gloria. Comunicación efectiva en el aula: técnicas de expresión oral para docentes. Barcelona: Graó, 2005.

SCHUTT, Russell K. Investigating the social world: the process and practice of research. Thousand Oaks: Sage, 2001.

SCOTTISH OFFICE. Review of Initial Teacher Education Stage 2: Review of the Review Group, 2005. Disponible en: <http://www.scotland.gov.uk/ Publications/2005/05/26142053/20548>. Acceso en: 27 nov. 2009.

TAGLIACARNE, Guglielmo. Técnica y práctica de las investigaciones de mercado. Barcelona: Ariel, 1968.

TEJADA, José. El trabajo por competencias en el prácticum: cómo organizarlo y cómo evaluarlo. Revista Electrónica de Investigación Educativa, México, v. 7, n. 2, p. 1-31, 2005. Disponible en: <http://redie.uabc.mx/vo7no2/contenido-tejada. html>. Acceso en: 29 febr. 2012.

VILLALOBOS, Ximena. Reflexión en torno a la gestión de aula y a la mejora en los procesos de enseñanza y aprendizajes. Revista Iberoamericana de Educación, OEI, n. 55/3, 2011. Disponible en: <http://www.rieoei.org/jano/4048Villalobos_Jano. pdf>. Acceso en: 20 febr. 2012.

Endereço para correspondência

Facultad de Ciencias de la Educación.

Campus universitario de Cartuja, s/n. Universidad de Granada.

18071. Granada (España)

Recebido em: 25-09-2013

Aprovado em: 19-02-2014

Movimento, Porto Alegre, v. 20, n. 2, p. 425-444, abr./jun. de 2014. 